\title{
COMPOSICIÓN QUÍMICA DEL AROMA DE TALLOS Y FRUTOS DE Rhipsalis baccifera (J. Miller) Stearn
}

\author{
CHEMICAL COMPOSITION OF SCENT FROM STEMS AND \\ FRUITS OF Rhipsalis baccifera (J. Miller) Stearn
}

\section{Adriana Bautista-San Juan', Juan Cibrián-Tovar ${ }^{2}$, Luis F. Salomé-Abarca ${ }^{2}$, R. Marcos Soto-Hernández ${ }^{3}$ y Emigdio De la Cruz-De la Cruz ${ }^{1}$}

\begin{abstract}
'Universidad Tecnológica de la Huasteca Hidalguense, Ing. en Biotecnología. Carr. Huejutla-Chalahuiyapa S/N. 43000, Colonia Tepoxteco, Tel. 01789-89-3-31-30. ²Postgrado de Entomología y Acarología, ${ }^{3}$ Postgrado de Botánica, Campus Montecillo, Colegio de Postgraduados. Km. 36.5 Carr. México-Texcoco. 56230, Col. Montecillos.
\end{abstract}

*Autor para correspondencia: (luis.salome@colpos.mx)

\section{RESUMEN}

Rhipsalis baccifera es una planta epífita que pertenece a la familia de las cactáceas. En México se usa para tratar problemas de salud como pérdida de cabello, diabetes y fracturas. No existen investigaciones sobre su actividad biológica. El objetivo de este estudio fue caracterizar la fracción aromática de $R$. baccifera. Los compuestos no volátiles y volátiles de $R$. baccifera se aislaron por extracción en disolvente orgánico, hidrodestilación, aireación dinámica y microextracción en fase sólida, y se analizaron por cromatografía de gases acoplada a espectrometría de masas. Se encontraron metabolitos secundarios como taninos, alcaloides y saponinas los cuales presentaron diferentes ubicaciones entre órganos. El análisis del extracto obtenido por hidrodestilación mostró la presencia de aldehídos, alcoholes, cetonas, monoterpenos, ácidos y alcanos. La aireación dinámica demostró que la composición volátil de tallos y frutos es diferente entre ambos órganos. Con esta técnica se detectaron metabolitos tales como cetonas, alcoholes, alcanos, monoterpenos, compuestos aromáticos y un éster. Incluso con variaciones en sus composiciones específicas, en ambos órganos, el D-limoneno fue el componente principal de la composición de ambos órganos, con 47.34 y 18.8 $\%$, respectivamente. $R$. baccifera tiene metabolitos secundarios que pueden sustentar su gama de actividades biológicas.

Palabras clave: Fracción volátil, aireación dinámica, D-limoneno, (Z)3-hexenol.

\section{SUMMARY}

Rhipsalis baccifera is an epiphytic plant from the Cactus family. In Mexico it is used to treat health problems such as hair loss, diabetes and fractures; however, there is no research on its biological activity. The non-volatile and volatile compounds were separated by organic extraction, hydro distillation, dynamic aeration and solid phase micro extraction. The volatile fraction of $R$. baccifera was analyzed by gas chromatography coupled to mass spectrometry. Secondary metabolites such as tannins, alkaloids and saponins were identified and localized to among plant organs. The extract obtained by hydro distillation contained aldehydes, alcohols, ketones, monoterpenes, acids and alkanes. Dynamic aeration showed that the chemical composition of the volatile fraction of stems and fruits is different between the two organs. By this technique, metabolites such as ketones, alcohols, alkanes, monoterpenes, aromatic compounds and an ester were detected. Even with variation in their specific compositions, D-limonene was the major component in both organs with 47.34 and $18.8 \%$ in stems and fruits respectively. $R$. baccifera has secondary metabolites that can sustain its broad range of biological activities.
Index words: Volatile fraction, dynamic aeration, D-limonene, (Z)-3hexenol.

\section{INTRODUCCIÓN}

Rhipsalis baccifera, comúnmente conocida como cactus muérdago en América Central y del Sur, el Caribe y Florida, es un cactus epífito que tiene su origen en las regiones antes mencionadas. También se propaga a lo largo de los trópicos del África y Sri Lanka (Anderson, 2001). Sus tallos son alargados-cilíndricos, y sus frutos son globosos, blanquecinos y transparentes cuando alcanzan la madurez. En México esta planta se usa en el tratamiento de fracturas, mediante un emplasto elaborado con el fruto y acompañado de la raíz macerada del bejuco rojo (Lonicera pilosa); previo a la aplicación del remedio, el curandero realiza las maniobras destinadas al arreglo de los huesos (Biblioteca Digital de la Medicina Tradicional Mexicana, 2009). Se usa también en el tratamiento de diabetes (Hernández-Galicia et al., 2002), para promover el crecimiento del cabello y para el fortalecimiento de la flora intestinal; además, tiene efectos contra Trichomonas vaginalis (Costa et al., 2013).

En raíces y tallos se ha detectado la presencia de alcaloides y saponinas. En cuanto a la fracción volátil, existe sólo un estudio realizado en la especie $R$. juengeri en el cual únicamente se ha caracterizado la fracción perteneciente a los frutos, donde undecan-2-ona y 10-metilundecan-2-ona son los compuestos más abundantes en esta fracción los cuales forman el 26.95 y $36.02 \%$ de su composición, respectivamente (Schlumpberger et al., 2006). Debido a los diversos usos medicinales que tiene R. baccifera, es importante hacer una caracterización fitoquímica que sustente científicamente el uso medicinal de esta planta y que abra la puerta hacia estudios de actividad biológica. Por lo tanto, el objetivo de esta investigación fue hacer un análisis preliminar de metabolitos secundarios y 
caracterización química de la fracción volátil de los tallos y frutos de R. baccifera.

\section{MATERIALES Y MÉTODOS}

\section{Material Vegetal}

Consistió de tallos ( $1.5 \mathrm{~m}$ de longitud) y frutos maduros de $R$. baccifera de los que se reunieron $5 \mathrm{~kg}$ de tallos y 300 $\mathrm{g}$ de frutos frescos, colectados en el municipio de Huejutla de Reyes, Hidalgo, México ( $21^{\circ} 09^{\prime} 13.1^{\prime \prime} \mathrm{N}$ y $98^{\circ} 23^{\prime} 06.7^{\prime \prime}$ O a $140 \mathrm{msnm}$ ) en enero de 2015. El material vegetal fue transportado al Colegio de Postgraduados-Campus Montecillo para su procesamiento e identificación, realizada por el M.C. Ricardo Vega Muñoz y confirmado por el Dr. Stephen D. Koch en enero del 2015. Un ejemplar fue depositado en el Herbario-hortorio CHAPA de esta institución.

\section{Extracción en disolvente orgánico}

En matraces Erlenmeyer de $200 \mathrm{~mL}$ se colocaron de manera independiente $10 \mathrm{~g}$ de material vegetal fresco, $10 \mathrm{~g}$ de material vegetal secado a $40^{\circ} \mathrm{C}$ y $10 \mathrm{~g}$ de material vegetal secado a $60^{\circ} \mathrm{C}$. A cada uno de los lotes $(n=3)$ se agregaron $60 \mathrm{~mL}$ de disolvente en el siguiente orden: agua:metanol en proporción 70:30, metanol, y diclorometano o hexano para obtener por separado los correspondientes extractos. Se dejaron extraer en cada caso por $72 \mathrm{~h}$ en obscuridad a temperatura ambiente. Al cumplir el tiempo de extracción, cada extracto fue filtrado y concentrado en un rotavapor Büchi B-480® (Flawil, Switzerland) con vacío, a $40^{\circ} \mathrm{C}$ hasta $10 \mathrm{~mL}$. Los extractos fueron almacenados a $-20^{\circ} \mathrm{C}$ hasta el análisis preliminar de metabolitos secundarios y cromatografía de capa fina.

\section{Análisis preliminar de metabolitos secundarios}

Flavonoides. Un mililitro de extracto se usó para la prueba de Shinoda, donde se agregó limadura de magnesio a cada extracto $(0.5 \mathrm{~g})$ y se agregaron cinco gotas de ácido clorhídrico concentrado. La formación de espuma roja indicó una reacción positiva (Murillo y Méndez, 2007); para esta prueba se utilizaron los extractos metanólico y de metanol:agua $(n=3)$.

Taninos. Se tomaron $2 \mathrm{~mL}$ de cada extracto y se agregaron cinco gotas de cloruro férrico al $5 \%$ en cada extracto por separado. La aparición de una coloración café-verdosa o azul obscuro fueron indicativos de una reacción positiva; para esta prueba se utilizaron los extractos metanólico y de metanol:agua $(n=3)$.
Saponinas. Se tomaron $2 \mathrm{~mL}$ de cada extracto y se agregaron 3 gotas de aceite mineral, posteriormente se agitaron vigorosamente por $3 \mathrm{~min}$. La formación de espuma y su permanencia por más de 15 min fue indicativa de una reacción positiva; para esta prueba se utilizaron los extractos de metanol:agua $(n=3)$.

Alcaloides. Se utilizaron $2 \mathrm{~mL}$ de cada extracto a los cuales se agregaron 5 gotas de reactivo de Dragendorff. La formación de una solución turbia color marrón fue indicativa de una reacción positiva; para esta prueba se utilizaron los extractos metanólicos $(n=3)$.

\section{Hidrodestilación}

Se hizo con un sistema tipo Clevenger, donde el material vegetal (96 g de tallos frescos) se colocó en un matraz de $2 \mathrm{~L}$ con fondo redondo y se agregaron $500 \mathrm{~mL}$ de agua destilada, se llevó a punto de ebullición durante $3 \mathrm{~h}$ contabilizadas a partir del inicio de la misma. Transcurrido este tiempo, se recuperó el hidrolato y éste se extrajo con diclorometano $(20 \mathrm{~mL})$ por tres veces; posteriormente se concentró en un rotavapor Büchi B-480® a $40{ }^{\circ} \mathrm{C}$, hasta eliminar la mayor cantidad de disolvente posible; el extracto aromático que quedó depositado en el matraz se transfirió a un vial de $1.5 \mathrm{~mL}$ con una pipeta Pasteur y se dejó evaporar el resto del solvente a temperatura ambiente en una campana de extracción. El método se realizó en tres repeticiones.

\section{Aireación dinámica}

Se colocaron de manera independiente $73 \mathrm{~g}$ de tallos frescos y $7 \mathrm{~g}$ de frutos frescos dentro de botellas de lavado de gas de $500 \mathrm{~mL}$ de volumen (ST/NS 40/50 Pyrex®), a través del cual se hizo pasar una corriente de aire filtrado con un cartucho que contenía 150 mg de Tenax®; el aire fue impulsado con una bomba de aire Elite 800 y dosificado con un flujometro (Gilmont $\AA$, modelo GS-8341-1201, Illinois, USA) a flujo de $500 \mathrm{~mL} \mathrm{~min}^{-1}$. Los volátiles provenientes del material vegetal fueron capturados en un segundo cartucho al final del sistema que también contenía 150 mg de absorbente. El tiempo de captura de volátiles fue de $24 \mathrm{~h}$. Al finalizar este tiempo el cartucho fue retirado y lavado con $6 \mathrm{~mL}$ de hexano y este disolvente fue colectado en un vial de $10 \mathrm{~mL}$. Posteriormente, el extracto hexánico fue concentrado bajo una corriente suave de nitrógeno hasta $1 \mathrm{~mL}$; finalmente, de este volumen se tomó $1 \mu \mathrm{L}$ para ser analizado por cromatografía de gases acoplada a espectrometría de masas. Como testigo se usó un sistema sin material biológico. Se hicieron tres repeticiones para este experimento. 


\section{Microextracción en fase sólida (MEFS)}

Se usaron viales de $15 \mathrm{~mL}$ de cristal ámbar, los cuales fueron lavados en la siguiente secuencia: Extran ${ }^{\circledR}$, agua, acetona, diclorometano y hexano, posteriormente fueron calentados a $300{ }^{\circ} \mathrm{C}$ por $3 \mathrm{~h}$ para eliminar presencia de compuestos volátiles del ambiente (Pineda-Ríos et al. 2016). Una vez listo el material (tallos y frutos) se colocaron $2 \mathrm{~g}$ de material vegetal fresco por separado, y el vial fue cerrado con un tapón de caucho tipo septa con línea de teflón y sellado con crimpers (Chromacol 20-ACB, 20 mm) marca Sigma Aldrich ${ }^{\circledR}$ (St. Louis, Missouri, USA). Para hacer la captura de volátiles se introdujo una fibra de MEFS de 50/30 $\mu \mathrm{m}$ de grosor de fase adsorbente, compuesta de divinilbenceno/carboxen/polidimetilsiloxano, marca Supelco®. El vial fue calentado a $50{ }^{\circ} \mathrm{C}$ en un plato térmico por 5 min, y posteriormente se esperó a alcanzar el equilibrio de la muestra por $15 \mathrm{~min}$. Una vez alcanzado el equilibrio se insertó la fibra en el vial. El tiempo de captura fue de 30 min para después ser aislada y analizada inmediatamente por cromatografía de gases. Como testigo se usó la fibra expuesta al interior de un vial vacío y sellado sin material biológico. Se hicieron tres repeticiones para este experimento.

\section{Cromatografía en capa fina (CCF)}

Se usaron placas cromatográficas de sílica gel $60 \mathrm{~F}_{254}$ (Merk KGaA®, Concord Road, USA) con soporte de aluminio, con medidas de $2 \times 6 \mathrm{~cm}$. Se aplicaron los extractos hexánicos y de diclorometano obtenidos por maceración en disolvente orgánico, se aplicó 1 mg de cada extracto. Para la separación se usó un sistema de elución basado en tolueno: acetato de etilo (93:7) y se reveló con vainillina al $1 \%$ en etanol y ácido sulfúrico al $10 \%$ en etanol, para posteriormente calentar la placa a $110^{\circ} \mathrm{C}$ por $5 \mathrm{~min}$.

\section{Cromatografía de gases acoplada a espectrometría de masas (CG-EM)}

Los extractos aromáticos y compuestos volátiles se analizaron mediante CG-EM con un cromatógrafo HP 6890 (Agilent Technologies $®$, USA) acoplado a un detector de masas 5973 (Agilent Technologies ${ }^{\circledR}$, USA). El sistema CG-EM usó una columna HP 5-M/ 30 m × $0.250 \mu$ m y 0.25 $\mu \mathrm{m}$ de grosor de fase estacionaria, como gas acarreador se usó helio con grado de pureza $99.9 \%$ a flujo de $1 \mathrm{~mL}$ $\mathrm{min}^{-1}$; la temperatura inicial en el horno fue de $40^{\circ} \mathrm{C}$ y aumentó $5{ }^{\circ} \mathrm{C} \mathrm{min}^{-1}$ hasta alcanzar una temperatura de 220 ${ }^{\circ} \mathrm{C}$; la temperatura del inyector en modo "splitless" (sin división de muestra) fue de $220^{\circ} \mathrm{C}$, y $280^{\circ} \mathrm{C}$ como temperatura de interfase. Para el detector de masas en modo SCAN, la temperatura de la fuente de iones fue de $230{ }^{\circ} \mathrm{C}$ y la del cuadrupolo de $250^{\circ} \mathrm{C}$. La energía de ionización fue de 70
eV. El volumen de inyección fue de $1 \mu \mathrm{L}$. Para la microextracción en fase sólida el tiempo de desorción de la fibra en el puerto de inyección a $240^{\circ} \mathrm{C}$ fue de $2 \mathrm{~min}$.

La identificación de los compuestos se hizo mediante la comparación de espectros de iones de las muestras con los de la biblioteca del Instituto Nacional de Estándares y Tecnología (NIST, por sus siglas en inglés) versión 2002, y se compararon tiempos de retención con los de estándares comerciales obtenidos de Sigma-Aldrich ${ }^{\circledR}$.

\section{RESULTADOS Y DISCUSIÓN}

El análisis preliminar mostró la presencia de taninos, saponinas y alcaloides en tallos (Cuadro 1), los frutos mostraron la misma tendencia en sus diferentes extractos, solo que, a diferencia de los tallos, no presentaron alcaloides, lo que coincide con lo reportado en la Biblioteca Digital de Medicina Tradicional Mexicana (2009). El hecho de que este material contenga tal tipo de metabolitos resalta su uso potencial como agente terapéutico, como en el caso de la tricomoniasis, donde el extracto de $R$. baccifera inhibe hasta $30 \%$ de la población parasítica durante una infección (Costa et al., 2013), y aunque no fue determinada la composición química exacta del extracto, es sabido que las saponinas pueden actuar como agentes antimicrobianos; por ejemplo, si se observan con el microscopio electrónico preparados de membranas lipídicas artificiales en presencia de saponinas, las membranas muestran grandes poros. Por lo tanto, se infiere que las saponinas forman complejos con los esteroles de las membranas celulares y producen grandes poros en las mismas que alteran su permeabilidad y la célula se lisa (Baumann et al., 2000).

Por otro lado, también presentaron taninos, los cuales, de acuerdo con Törrönen (2009), exhiben capacidad antioxidante, antimicrobiana, anti-inflamatoria y anticancerígena. Por otra parte, taninos como los elagitaninos pueden prevenir la obesidad (Xiang et al., 2008), lo cual podría sustentar el uso de esta planta como hipoglucemiante. La ingesta de alimentos ricos en elagitaninos puede mejorar la salud y prevenir enfermedades crónicas como enfermedades cardiovasculares, enfermedades neurodegenerativas y cáncer.

Los alcaloides también están presentes en esta planta; por lo general, estos compuestos tienen una gama notable de actividad farmacológica a menudo dramática, y también suelen ser tóxicos para el hombre (Talib and Mahasneh, 2010). Muchos alcaloides se utilizan en terapéutica como herramientas farmacológicas. Se ha documentado una amplia gama de efectos biológicos de alcaloides, que incluyen efectos eméticos, anticolinérgicos, antitumorales, diuréticos, simpático-miméticos, antivirales, antihipertensivos, 
Cuadro 1. Análisis preliminar de metabolitos secundarios de tallos de $R$. baccifera con extractos de diferentes polaridades.

\begin{tabular}{llcccc}
\hline Nivel de secado & Disolvente & Flavonoides & Taninos & Alcaloides & Saponinas \\
\hline $40^{\circ} \mathrm{C}$ & Metanol-agua (3:7) & - & + & - & ++ \\
& Metanol & - & - & + & - \\
& Diclorometano & - & - & - & - \\
\multirow{5}{*}{$60^{\circ} \mathrm{C}$} & Hexano & - & - & - & - \\
& Metanol-agua (3:7) & - & + & - & ++ \\
& Metanol & - & + & - & - \\
\multirow{5}{*}{ Fresco } & Diclorometano & - & - & - & - \\
& Hexano & - & - & - & + \\
& Metanol-agua (3:7) & - & + & - & - \\
& Metanol & - & - & - & - \\
& Diclorometano & - & - & - & - \\
\hline
\end{tabular}

+: concentración baja; ++: concentración media; -: no detectado.

hipno-analgésicos, antidepresivos, mio-relajante, antimicrobianos y antiinflamatorios (Aiello et al., 2011; Ezell et al., 2010; Henriques et al., 2004).

El análisis por cromatografía en capa fina (CCF) para terpenos mostró que el material seco ofrece una mejor extracción al obtener un mayor número de bandas que en el material fresco (Figura 1). Las relaciones de frente (Rfs) para las bandas obtenidas variaron de 0.04 a 0.87 (Cuadro 2). Aunque en el material secado a $40{ }^{\circ} \mathrm{C}$ y a $60^{\circ} \mathrm{C}$ se encontró el mismo número de bandas, éstas difieren en color y quizá se trate de diferentes compuestos. Por lo anterior, la extracción en disolvente orgánico debe hacerse en materiales procesados de distinta forma para ofrecer una caracterización completa de la fracción terpénica de R. baccifera.

Con la extracción hecha con hexano se observó un mayor número de bandas (Cuadro 3), lo cual indica que el hexano ofrece una mejor extracción que el diclorometano y la temperatura de secado a $60^{\circ} \mathrm{C}$ es mejor que la de 40 ${ }^{\circ} \mathrm{C}$. Lo anterior puede deberse que a mayor temperatura de secado se elimina más agua que puede estar interfiriendo con la extracción debido a que es de polaridad opuesta a la del hexano; aun así, cabe recordar que a mayor temperatura se corre el riesgo de perder compuestos volátiles.

Ambas técnicas, hidrodestilación y aireación dinámica, permitieron obtener compuestos volátiles de tallos y frutos de $R$. baccifera, los cuales fueron separados y detectados por cromatografía de gases acoplada a espectrometría de masas (CG-EM). Por la técnica de hidrodestilación se obtiene un mayor número de picos separados (Figuras 2 y 3 ), y por lo tanto, un mayor número de compuestos, aunque se pierden algunos compuestos de bajo peso molecular como el D-limoneno, el cual es posible de extraer a través de aireación dinámica.

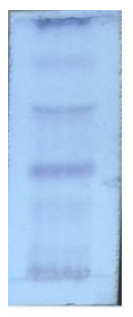

1

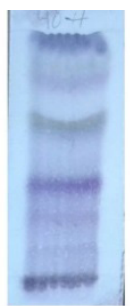

2

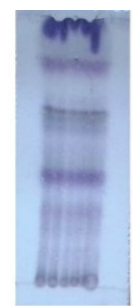

3

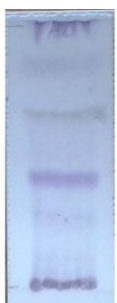

4

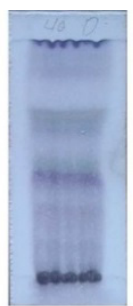

5

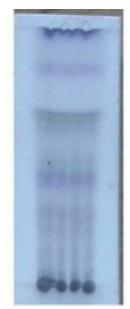

6

Figura 1. Cromatografía de capa fina de extractos hexánicos y diclorometano de tallos de R. baccifera. 1: Tallos frescos extraídos con hexano; 2: Tallos secados a $40^{\circ} \mathrm{C}$ y extraídos con hexano; 3: Tallos secados a $60^{\circ} \mathrm{C}$ y extraídos con hexano; 4: Tallos frescos extraídos con diclorometano; 5 : Tallos secados a $40^{\circ} \mathrm{C}$ y extraídos con diclorometano y 6 : Tallos secados a $60^{\circ} \mathrm{C}$ y extraídos con diclorometano. 
Cuadro 2. Número de bandas obtenidas de material vegetal fresco y secado a diferentes temperaturas extraído con diclorometano.

\begin{tabular}{ccccccc}
\hline \multirow{2}{*}{ Número de banda } & \multicolumn{2}{c}{ M. S. $40^{\circ} \mathrm{C}$} & \multicolumn{2}{c}{ M. S. $60{ }^{\circ} \mathrm{C}$} & \multicolumn{2}{c}{ Fresco } \\
\cline { 2 - 7 } & $\mathrm{Rf}^{\dagger}$ & Color de banda & $\mathrm{Rf}^{\dagger}$ & Color de banda & $\mathrm{Rf}^{\dagger}$ & Color de banda \\
\hline 1 & 0.04 & Violeta & 0.04 & Azul & 0.11 & Violeta \\
2 & 0.31 & Violeta & 0.11 & Violeta & 0.25 & Violeta \\
3 & 0.43 & Morado & 0.30 & Violeta & 0.41 & Morado \\
4 & 0.48 & Verde & 0.41 & Morado & 0.67 & Gris \\
5 & 0.60 & Morado & 0.51 & Verde & 0.86 & Morado \\
6 & 0.65 & Azul & 0.60 & Morado & - & - \\
7 & 0.70 & Gris & 0.65 & Verde & - & - \\
8 & 0.73 & Morado & 0.67 & Negro & - & - \\
9 & 0.87 & Morado & 0.86 & Morado & - & - \\
\hline
\end{tabular}

${ }^{\dagger}$ Rf: relación de frentes; M. S.: material secado.

Cuadro 3. Relación de frentes de bandas obtenidas de material vegetal fresco y secado a diferentes temperaturas extraído con hexano.

\begin{tabular}{ccccccc}
\hline \multirow{2}{*}{ Número de banda } & \multicolumn{2}{c}{ M. S. $40{ }^{\circ} \mathrm{C}$} & \multicolumn{2}{c}{ M. S. $60{ }^{\circ} \mathrm{C}$} & \multicolumn{2}{c}{ Fresco } \\
\cline { 2 - 7 } & $\mathrm{Rf}^{\dagger}$ & Color de banda & $\mathrm{Rf}^{\dagger}$ & Color de banda & $\mathrm{Rf}^{\dagger}$ & Color de banda \\
\hline 1 & 0.14 & Violeta & 0.18 & Gris & 0.09 & Violeta \\
2 & 0.26 & Violeta & 0.27 & Violeta & 0.27 & Violeta \\
3 & 0.39 & Morado & 0.39 & Morado & 0.43 & Morado \\
4 & 0.58 & Violeta & 0.46 & Verde & 0.61 & Violeta \\
5 & 0.60 & Azul & 0.51 & Violeta & 0.65 & Azul \\
6 & 0.65 & Negro & 0.55 & Gris & 0.84 & Violeta \\
7 & 0.82 & Morado & 0.60 & Morado & - & - \\
8 & 0.87 & Verde & 0.65 & Azul & - & - \\
9 & - & - & 0.67 & Negro & - & - \\
10 & - & - & 0.72 & Violeta & - & - \\
11 & - & - & 0.83 & Morado & - & - \\
\hline
\end{tabular}

${ }^{\dagger}$ Rf: relación de frentes; M. S.: material secado.

A través del análisis por CG-EM del extracto aromático obtenido por hidrodestilación se detectó un total de 35 compuestos, de los cuales la mayoría son aldehídos; además, se observaron alcoholes, cetonas, monoterpenos, ácidos y alcanos. El compuesto mayoritario fue un alcohol, el (Z)-3-hexen-1-ol con contenido de $8.62 \%$ (Cuadro 4).

En relación con la fracción volátil colectada por aireación dinámica, el análisis por CG-EM mostró la presencia de cetonas, alcoholes, alcanos, monoterpenos, compuestos aromáticos y un éster. El compuesto que se encontró en mayor proporción fue D-limoneno con 47.34 \% (Cuadro 5).

En cuanto a la composición de la fracción volátil en frutos, al igual que en tallos, fue el D-limoneno el componente mayoritario con 18.8 \% de composición además de otro monoterpeno, un sesquiterpeno y alcanos en su mayoría (Cuadro 6). Se puede observar que la proporción del D-limoneno no es constante en los diferentes órganos, puesto que se encuentra aproximadamente tres veces más elevada en tallo que en frutos.

Por otro lado, el D-limoneno también fue aislado de la fracción volátil de Rhipsalis juengeri, aunque como compuesto traza $(<0.001 \%)$, y fueron undecan-2-ona y 10-metilundecan-2-ona los compuestos más abundantes en esta fracción, los cuales conforman el 26.95 y 36.02 \%, respectivamente (Schlumpberger et al., 2006). De esta forma, se puede decir que el aroma entre especies de Rhipsalis varía y está determinado por diferentes grupos químicos, y son 


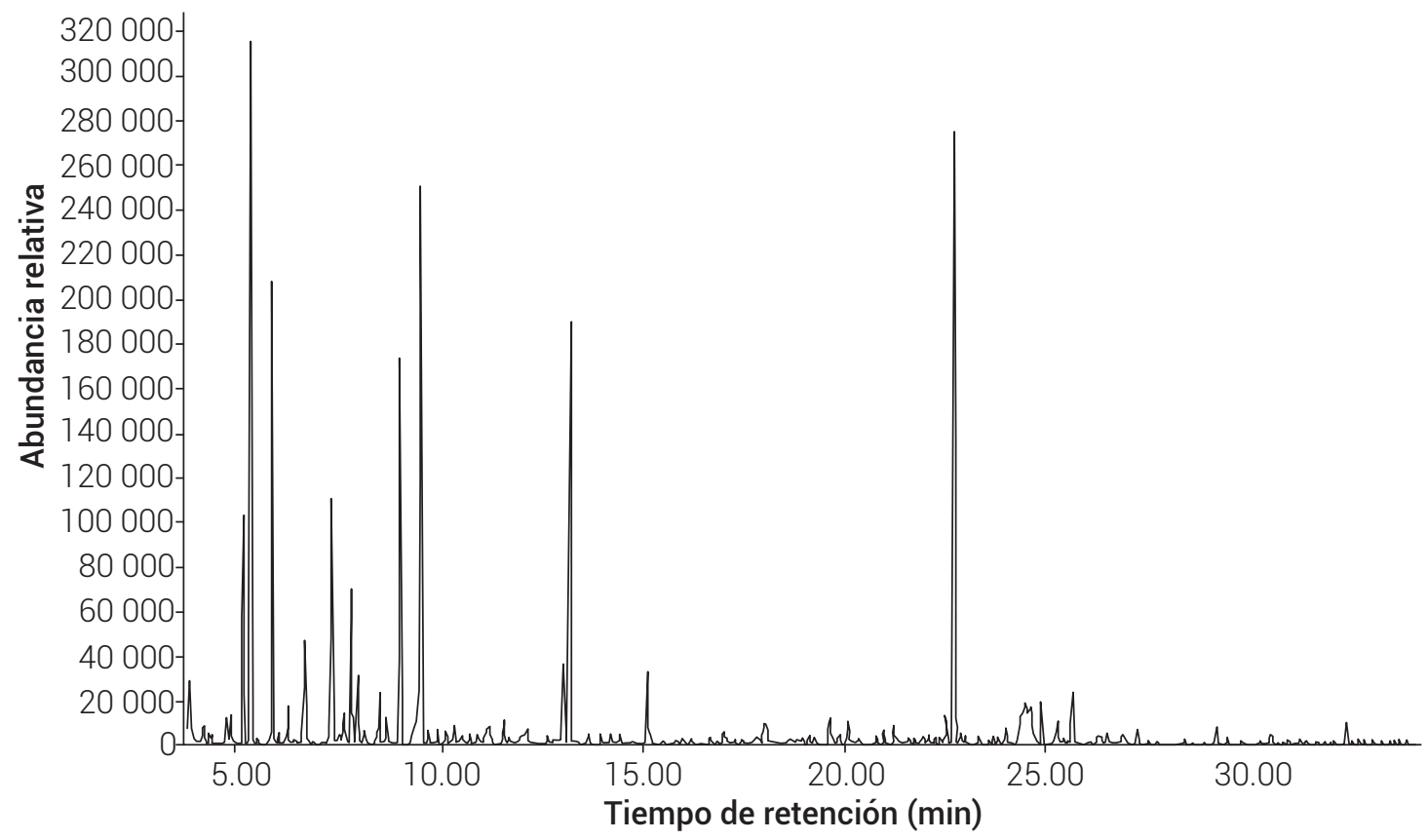

Figura 2. Cromatograma de la fracción volátil de tallos de $R$. baccifera.

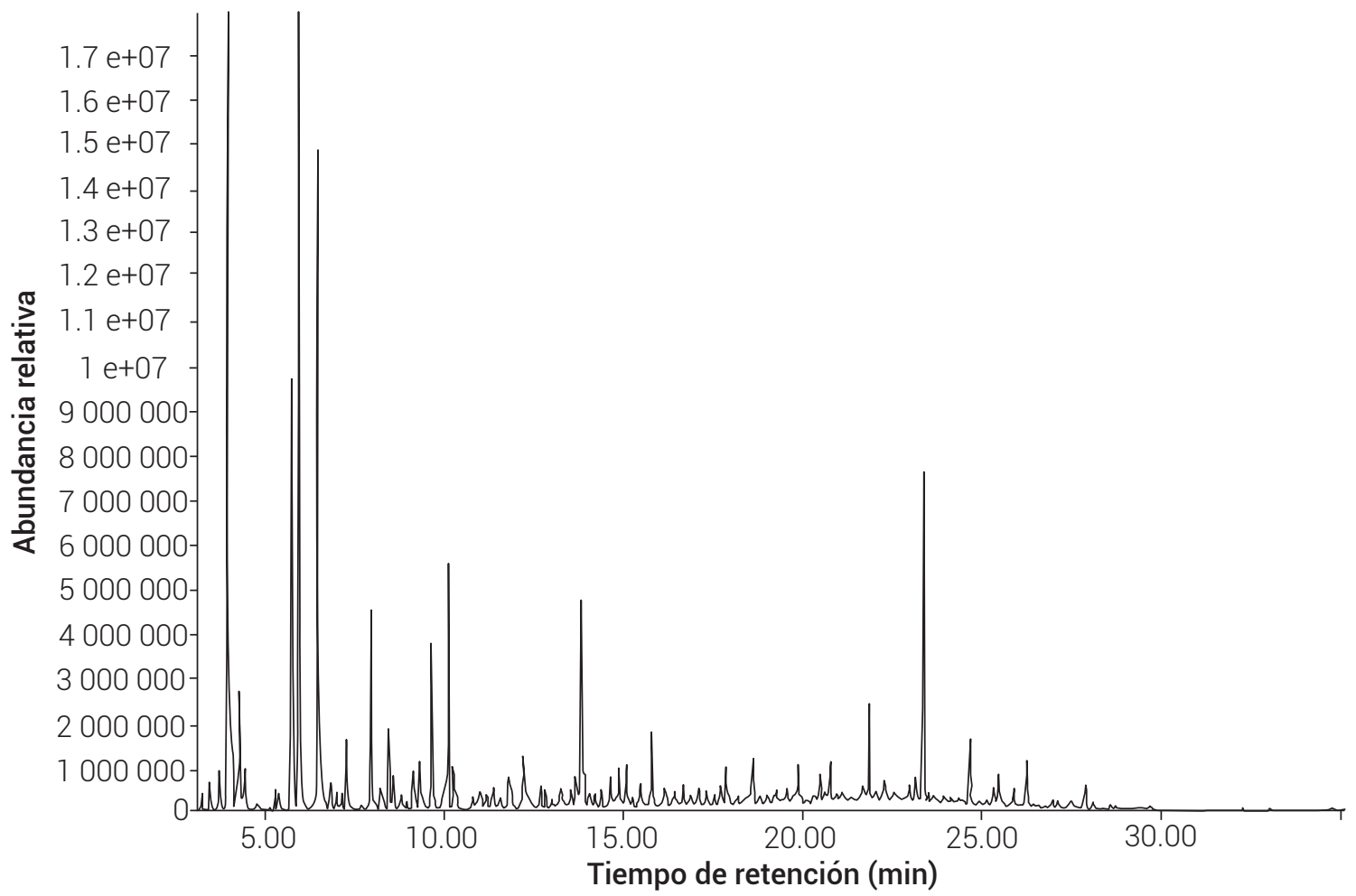

Figura 3. Cromatograma de la fracción volátil de frutos de $R$. baccifera.

las cetonas las que determinaron el aroma para R. jungeri, y para $R$. baccifera el grupo de monoterpenos los que determinaron su olor. Además, R. jungeri presenta $28 \mathrm{com}-$ puestos más que $R$. baccifera, lo cual sugiere que la composición volátil entre especies de la misma familia varía no sólo en las concentraciones en que se encuentran dentro de la fracción sino en el número de compuestos presentes.

La función del D-limoneno en esta planta podría ser de tipo ecológico, pues según Theis y Lerdau (2003), los terpenos pueden servir a una variedad de funciones, que incluyen disuasión del herbívoro, toxicidad contra hongos y 
Cuadro 4. Fracción aromática de tallos de $R$. baccifera obtenida por hidrodestilación.

\begin{tabular}{|c|c|c|c|}
\hline Compuesto & $\operatorname{TR}(\min )$ & Porcentaje de Identificación & Composición (\%) \\
\hline Hexanal $^{+}$ & 3.92 & 90 & 3.06 \\
\hline Furfural & 4.56 & 83 & 2.27 \\
\hline (E)-2-Hexanal & 4.97 & 94 & 0.62 \\
\hline (Z)-3-Hexen-1-o1 ${ }^{+}$ & 5.03 & 91 & 8.62 \\
\hline (E)-2-Hexen-1-o1 & 5.27 & 91 & 0.99 \\
\hline Hexil alcohol & 5.32 & 64 & 4.47 \\
\hline Butilacetona & 5.83 & 64 & 0.61 \\
\hline Heptaldehído & 6.08 & 90 & 1.04 \\
\hline Prenal & 6.47 & 53 & 1.38 \\
\hline 1-Metil-2-propilciclohexano & 7.27 & 64 & 1.39 \\
\hline (E)-2-Heptanal & 7.49 & 83 & 4.52 \\
\hline Ácido butoxiacético & 8.13 & 47 & 1.32 \\
\hline Ácido caproico & 8.25 & 72 & 2.07 \\
\hline Octan-2-ona & 8.46 & 80 & 0.78 \\
\hline 2,4-Heptadien-1-al & 8.61 & 90 & 0.53 \\
\hline Caprilaldehído & 8.78 & 86 & 1.48 \\
\hline 1,1- Dietoxihexano & 9.43 & 45 & 0.48 \\
\hline a- Tolualdehído & 9.93 & 91 & 3.31 \\
\hline (E)-2-Tridecenal & 10.35 & 64 & 1.73 \\
\hline Pentilciclopropano & 10.72 & 96 & 1.79 \\
\hline Ácido enántico & 10.92 & 72 & 1.04 \\
\hline Linalol $^{+}$ & 11.56 & 83 & 0.68 \\
\hline Nonaldehído & 11.68 & 86 & 3.74 \\
\hline (E)-2-Nonenal & 13.27 & 86 & 0.83 \\
\hline Ácido caprílico & 13.78 & 86 & 4.92 \\
\hline Terpineol $^{+}$ & 14.18 & 91 & 1.26 \\
\hline 2,4-Nonadien-1-al & 14.80 & 81 & 0.47 \\
\hline Benzosulfonazole & 15.08 & 81 & 0.96 \\
\hline 2-Borneno & 15.24 & 93 & 1.86 \\
\hline 2-Metileno-ciclopentanol & 16.12 & 53 & 2.71 \\
\hline p-Vinilguaiacol & 17.56 & 90 & 1.28 \\
\hline$(E, E)$-2,4-Decadienal & 17.60 & 93 & 2.35 \\
\hline
\end{tabular}

${ }^{\dagger}$ Indica compuestos cuya identidad fue corroborada con estándares; TR: tiempo de retención.

atracción de polinizadores. Los terpenoides involucrados con la atracción de polinizadores tienden a estar situados en partes de la planta donde la resistencia al flujo es muy baja, y las tasas de flujo sustanciales pueden ocurrir incluso a bajas concentraciones, del orden de microgramos por gramo (Ayasse et al., 2000). Por otro lado, los tallos de $R$. baccifera, al presentar una considerable fracción de D-limoneno, podrían ser probados en otros tipos de enfermedades en las cuales dicho compuesto tiene efectos po- sitivos como en el tratamiento de cálculos biliares, donde el D-limoneno se ha utilizado clínicamente para disolver aquellos que contienen colesterol.

Este compuesto también se ha utilizado para el tratamiento de la acidez estomacal, debido a su efecto de neutralización de ácido gástrico y la mejora de la peristalsis. Además, la actividad de este compuesto ha sido establecida como quimiopreventiva contra muchos tipos de cánceres. 
Cuadro 5 . Compuestos volátiles aislados de tallos de $R$. baccifera por aireación dinámica.

\begin{tabular}{|c|c|c|c|}
\hline Compuesto & $\mathrm{TR}(\min )$ & Porcentaje de identificación & Composición (\%) \\
\hline 3-Hexanol ${ }^{+}$ & 3.83 & 78 & 2.59 \\
\hline 3-Metilciclopentanona & 4.84 & 90 & 1.66 \\
\hline Acetato de metoxi propanol & 5.36 & 78 & 4.31 \\
\hline 5-Metoxi-2-metil-2-pentanol & 6.25 & 42 & 2.56 \\
\hline 2-Metil-4-heptanona & 6.59 & 72 & 1.65 \\
\hline 1-Metil-ciclopentanol & 7.81 & 49 & 11.46 \\
\hline Decano & 8.67 & 87 & 1.90 \\
\hline 1,2-dietil-Benceno & 9.30 & 46 & 1.56 \\
\hline D-limoneno $^{+}$ & 9.49 & 96 & 47.34 \\
\hline 2-Metiletilhexanoato & 9.92 & 45 & 1.54 \\
\hline a-Terpineno ${ }^{+}$ & 11.22 & 93 & 1.68 \\
\hline Undecano & 11.54 & 72 & 1.77 \\
\hline Acido 2-Etilhexilester acético & 13.02 & 78 & 6.51 \\
\hline Benzotiazol & 15.08 & 90 & 6.79 \\
\hline a-Bergamoteno & 19.57 & 59 & 2.45 \\
\hline$(+)$-Longifoleno & 20.00 & 95 & 1.83 \\
\hline 5,6-Dipropil-decano & 22.44 & 59 & 2.39 \\
\hline
\end{tabular}

†'Indica compuestos cuya identidad fue corroborada con estándares; TR: tiempo de retención.

Cuadro 6. Compuestos volátiles de frutos de $R$. baccifera aislados por aireación dinámica.

\begin{tabular}{lccc}
\hline Compuesto & TR $(\mathrm{min})$ & Porcentaje de identificación & Composición (\%) \\
\hline Nonano & 6.622 & 95 & 5.8 \\
3-Metil-1-penten-3-ol $^{\text {Decano }}$ & 7.956 & 64 & 14.6 \\
D-limoneno $^{+}$ & 9.374 & 97 & 3.2 \\
Undecano $^{+}$ & 10.162 & 92 & 18.8 \\
a-Terpineol $^{+}$ & 12.207 & 95 & 2.4 \\
Dodecano $_{\text {Isobutil isobutirato }}$ & 14.869 & 91 & 3.2 \\
D-longifoleno $^{\text {Pentadecano }}$ & 15.071 & 96 & 4.3 \\
Hexadecano & 19.849 & 47 & 3.4 \\
Heptadecano & 20.762 & 99 & 3.6 \\
Octadecano & 22.989 & 96 & 5.8 \\
\hline
\end{tabular}

†Indica compuestos cuya identidad fue corroborada con estándares; TR: tiempo de retención.

La evidencia de una fase I de ensayos clínicos muestra una respuesta parcial en un paciente con cáncer de mama y enfermedad estable durante más de seis meses en tres pacientes con cáncer colorrectal (Sun, 2007).

En cuanto a la identificación del D-limoneno, además de comparar su tiempo de retención con el de un estándar, se hizo la comparación de espectros de iones (Figura 4), donde se puede observar la correspondencia de los iones mayoritarios para ambos espectros, con excepción del ion 57 el cual sí está presente en el espectro de la biblioteca pero con menor intensidad, probablemente por un fenómeno de 
isotopía; por otro lado, el pico base para ambos espectros es el ion con masa 68 y ambos presentan abundancia de 100; aunque no se formó el ion molecular, se observa la presencia de la masa 136 para ambos espectros, la cual corresponde al peso molecular del D-limoneno.

Se identificaron 14 compuestos a través del análisis cromatográfico de los olores aislados con MEFS a través de microextracción en fase sólida, de los cuales $28.57 \%$ son sesquiterpenos, $21.43 \%$ monoterpenos, $21.43 \%$ aldehídos,
$14.29 \%$ alcoholes y el resto conformado por una furanocumarina y una cetona. El compuesto mayoritario fue (Z)-3hexen-1-ol, el cual también es el compuesto mayoritario obtenido por hidrodestilación y aislado en tallos (Cuadro 7).

Para el caso de la fracción volátil analizada por MEFS en frutos se detectaron sólo nueve compuestos también conformados por cetonas, aldehídos, alcoholes, sesqui y monoterpenos (Cuadro 8).

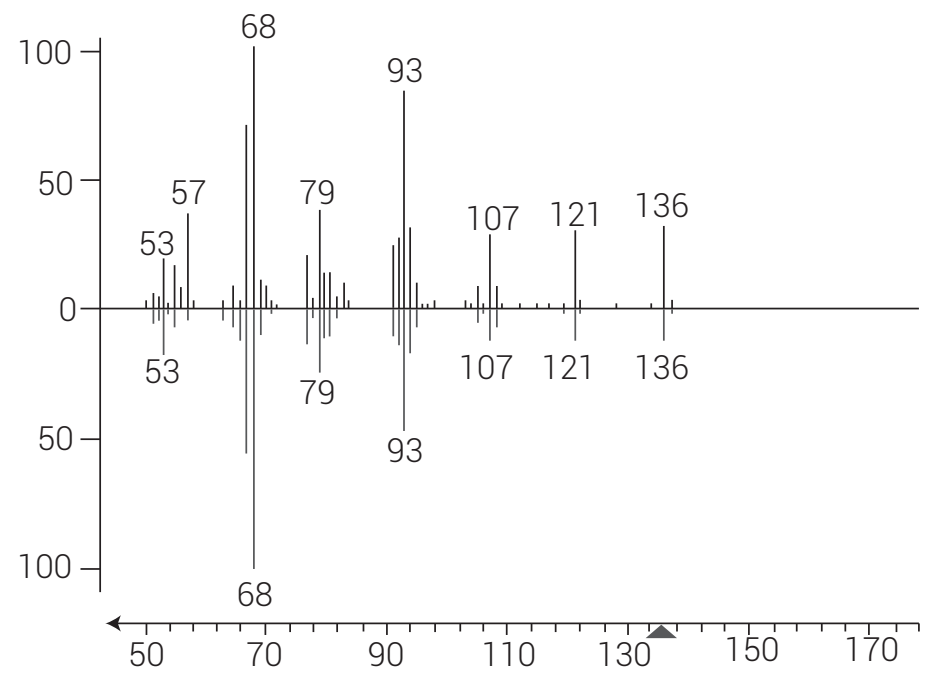

Figura 4. Comparación del espectro del D-limoneno de la muestra (espectro superior) y el proveniente de la biblioteca NIST (espectro inferior).

Cuadro 7. Compuestos volátiles de tallos de $R$. baccifera aislados con microextración en fase sólida.

\begin{tabular}{lccc}
\hline Compuesto & TR $(\mathrm{min})$ & Porcentaje de identificación & Composición (\%) \\
\hline Hexanal $^{+}$ & 4.3 & 72 & 13.9 \\
2-Hexenal & 5.42 & 95 & 15.4 \\
Z)-3-Hexen-1-ol $^{+}$ & 5.49 & 96 & 56.4 \\
Felandreno $^{+}$ & 8.55 & 68 & 0.4 \\
1-Octen-3-ol & 8.71 & 94 & 3.2 \\
3-Octanona & 8.91 & 94 & 2.8 \\
Eucaliptol $^{+}$ & 10.22 & 62 & 0.4 \\
Pineno $^{+}$ & 10.39 & 90 & 0.3 \\
Nonanal & 12.32 & 83 & 2.9 \\
Cubebeno $_{\text {Bourboneno }}$ & 19.95 & 83 & 0.5 \\
Bergamoteno & 20.19 & 86 & 1.0 \\
Calareno & 20.28 & 72 & 2.2 \\
Cadineno & 21.07 & 83 & 0.4 \\
\hline
\end{tabular}

${ }^{\top}$ Indica compuestos cuya identidad fue corroborada con estándares; TR: tiempo de retención. 
Cuadro 8. Compuestos volátiles de frutos de $R$. baccifera aislados con microextracción en fase sólida.

\begin{tabular}{lccc}
\hline Compuesto & TR $(\mathrm{min})$ & Composición (\%) & Porcentaje de identificación \\
\hline 3-Hexanol & 4.24 & 4.4 & 84 \\
Hexanal $^{+}$ & 4.33 & 12.2 & 83 \\
Pineno $^{+}$ & 7.45 & 9.8 & 94 \\
Careno $_{\text {1-Octen-3-ol }}$ & 8.64 & 2.1 & 76 \\
3-Octanona & 8.7 & 36.9 & 86 \\
3-Octanol & 8.92 & 24.7 & 95 \\
Eucaliptol $^{+}$ & 9.17 & 2.3 & 80 \\
Nonanal & 10.21 & 3.5 & 91 \\
\hline
\end{tabular}

†Indica compuestos cuya identidad fue corroborada con estándares; TR: tiempo de retención.

\section{CONCLUSIONES}

R. baccifera presenta taninos, terpenos, saponinas y alcaloides en tallos, y sólo taninos, terpenos y saponinas en frutos. El material secado a $60{ }^{\circ} \mathrm{C}$ extraído con hexano ofrece una más rápida y mejor caracterización por cromatografía en capa fina de la fracción terpénica de tallos de $R$. baccifera. Existen diferencias de composición entre la fracción semivolátil obtenida por hidrodestilación y la volátil obtenida por aireación dinámica, donde resultó el 3-hexenol el compuesto mayoritario en la semivolátil y el D-limoneno en la fracción volátil. El D-limoneno es el metabolito más abundante en tallos y frutos de R. baccifera con 47.34 y $18.8 \%$, respectivamente. Las diferencias entre la composición de tallo y fruto son en número, tipo y proporciones de los metabolitos volátiles que las componen. $R$. baccifera presenta metabolitos secundarios que sustentan sus diversos usos terapéuticos. Con base en las tres técnicas de aislamiento se concluye que el aroma de $R$. baccifera está determinado en su mayoría por monoterpenos y alcoholes.

\section{BIBLIOGRAFÍA}

Aiello A., E. Fattorusso, C. Imperatore, C. Irace, P. Luciano, M. Menna, R. Santamaria and R. Vitalone (2011) Zorrimidazolone, a bioactive alkaloid from the non-indigenous Mediterranean stolidobranch Polyandrocarpa zorritensis. Marine Drugs 9:1157-1165.

Anderson E. F. (2001) The Cactus Family. Timber Press. Portland, Oregon. $776 \mathrm{p}$.

Ayasse M., F. P. Schiestl, H. F. Paulus, C. Löfstedt, B. Hansson, F. Ibarra and W. Francke (2000) Evolution of reproductive strategies in the sexually deceptive orchid Ophrys sphegodes: how does flower-specific variation of odor signals influence reproductive success? Evolution 54:1995-2006.

Baumann E., G. Stoya, A. Völkner, W. Richter, C. Lemke and W. Linss (2000) Hemolysis of human erythrocytes with saponin affects the membrane structure. Acta Histochemica 102:21-35.

Biblioteca Digital de la Medicina Tradicional Mexicana (2009) Atlas de las Plantas de la Medicina Tradicional Mexicana. http:// www.medicinatradicionalmexicana.unam.mx/monografia. php?|=3\&t=Rhipsalis\%20baccifera\&id=7389. (Abril 2015).

Costa B. C. L., P. de Brum V., A. J. Macedo and T. Tasca (2013) Remarkable anti-trichomonas vaginalis activity of plants traditionally used by the Mbyá-Guarani indigenous group in Brazil. BioMed Research International 826370. http://dx.doi.org/10.1155/2013/826370.

Ezell S. J., H. Li, H. Xu, X. Zhang, E. Gurpinar, X. Zhang, E. R. Rayburn, C. I. Sommers, X. Yang, S. E. Velu, W. Wang and R. Zhang (2010) Preclinical pharmacology of BA-TPQ, a novel synthetic iminoquinone anticancer agent. Marine Drugs 8:2129-2141.

Henriques A. T., R. P. Limberger, V. A. Kerber e P. R. H. Moreno (2004) Alcaloides: generalidades e aspectos básicos: In: Farmacognosia da Planta ao Medicamento. $5^{\text {a }}$ ed. C. M. O. Simões, E. P. Schenkel, G. Gosmann, J. C. P. de Mello, L. A. Mentz e P. R. Petrovick (eds.). Editora da Universidade Federal de Santa Catarina. Florianópolis, Brasil. pp:765-792.

Hernández-Galicia E., A. Aguilar-Contreras, L. Aguilar-Santamaría, R. RománRamos, A. A. Chávez-Miranda, L. M. García-Vega, J. L. Flores-Sáenz and F. J. Alarcón-Aguilar (2002) Studies on hypoglycemic activity of mexican medicinal plants. Proceedings of the Western Pharmacology Society 45:118-124.

Murillo P. E. y J. J. Méndez A. (2007) Guía Metodológica para la Detección Rápida de Algunos Núcleos Secundarios. Manual de Fitoquímica. Universidad del Tolima. Ibagué, Colombia. 24 p.

Pineda-Ríos J. M., J. Cibrián T., J. Macías S., L. F. Salomé A., R. M. López R. y E. J. Arjona S. (2016) La composición y proporción de los componentes de la feromona sexual de Hypsipyla grandella Zeller (Lepidoptera: Pyralidae) varía dependiendo de la técnica utilizada para su aislamiento. Entomotrópica 31:172-185.

Schlumpberger B. 0., R. A. Clery and W. Barthlott (2006) A unique cactus with scented and possibly bat-dispersed fruits: Rhipsalis juengeri. Plant Biology 8:265-270.

Sun J. (2007) D-limonene: safety and clinical applications. Alternative Medicine Review 12:259-264.

Talib W. H. and A. M. Mahasneh (2010) Antimicrobial, cytotoxicity and phytochemical screening of Jordanian plants used in traditional medicine. Molecules 15:1811-1824.

Theis N. and M. Lerdau (2003) The evolution of function in plant secondary metabolites. International Journal of Plant Sciences 164:93-102

Törrönen A. R. (2009) Sources and health effects of dietary ellagitannins. In: Chemistry and Biology of Ellagitannins: An Underestimated Class of Bioactive Plant Polyphenols. S. Quideau (ed.). World Scientific Publishing. Toh Tuck Link, Singapore. pp:298-319.

Xiang L., D. Xing, F. Lei, W. Wang, L. Xu, L. Nie and L. Du (2008) Effects of season, variety, and processing method on ellagic acid content in pomegranate leaves. Tsinghua Science and Technology 13:460-465. 